\title{
Linear phase retrieval for real-time adaptive optics
}

A. Polo

\section{A. Haber}

S. F. Pereira

\section{Verhaegen}

Optics Research Group, Delft University of Technology, Delft, The Netherlands

Delft Center for Systems and Control, Delft University of Technology, Delft, The Netherlands

Optics Research Group, Delft University of Technology, Delft, The Netherlands

Delft Center for Systems and Control, Delft University of Technology, Delft, The Netherlands

\section{H. P. Urbach}

Optics Research Group, Delft University of Technology, Delft, The Netherlands

\begin{abstract}
We developed a fast phase retrieval algorithm that is suitable for real-time applications such as adaptive optics. The phase retrieval model is developed by linearising the pupil function in the approximation of small aberrations and is valid for low-NA focused field. The linear model in conjunction with a particular choice for the position of the single out-of-focus measurement plane and an efficient control algorithm, significantly reduces the computation time for phase retrieval. The experimental results demonstrate the validity of the described approach for fast correction of aberrations.
\end{abstract}

[DOI: http://dx.doi.org/10.2971/jeos.2013.13070]

Keywords: Phase retrieval, inverse problem, adaptive optics, phase measurement

\section{INTRODUCTION}

Applications of adaptive optics [1] (AO) are rapidly spreading in several fields outside of its initial use in astronomy. In the past decades a considerable amount of research has been done to extend its use in microscopy [2,3], ophthalmology [4], tomography [5], beam shaping [6] and even in the semiconductor industry $[7,8]$. The principle of an AO system is to correct wavefront aberrations in real-time using an active optical element based on a control algorithm and knowledge of the aberrations measured by a wavefront sensor (WFS). Nowadays, several types of WFSs are available and they are generally divided into two classes: direct wavefront sensors and indirect wavefront sensors [9]. The most used sensors, such as Shack-Hartmann wavefront sensors, lateral shear interferometers and curvature sensors belong to the first class in which the wavefront shape in the pupil of the system is determined using zonal or modal measurements. Indirect wavefront sensor $[10,11]$ are those in which one or more images of an object (usually a point source) are used as input for a phase retrieval algorithm which retrieves the aberrations in the pupil plane. Compared to the direct wavefront sensor, an indirect sensor requires little or no optics other than the imaging sensor. Moreover, an indirect wavefront sensor is fully sensitive to all aberrations up to the focal plane. However, the time required to estimate the aberrations is considerable longer than needed for a direct wavefront sensor. This is mainly caused by the necessity to measure several out-of-focus images in order to achieve a stable and accurate estimate of the aberrations [12]. An effective way to reduce the computational time of the aberration retrieval problem is by identifying a single plane of measurement in the focal region which is optimal for phase retrieval. Several works have been done in this direction such as Fienup [13], Lee [14] and El Gawhary [15]. In [13, 14] it has been shown that the optimal plane of measurement can be identified on the basis of the Cramer-Rao lower-bound. In [15] the authors use the definition of spatial correlations. Another approach is described in [16] where we used a small aberrations approximation to solve this problem.

The single-plane measurement approach benefits in real-time phase correction applications from the absence of moving parts in the setup and moreover from the minimum data load that is required to be analysed. Nevertheless a phase retrieval algorithm usually involves a non-linear inverse problem which is generally computationally complex and must be solved iteratively. Therefore, in order to obtain a real-time phase estimation based on non-linear phase retrieval, a highperformance computing system has to be employed to decrease the computational time required for the phase aberration estimation. When such hardware is not available, proper approximations of the phase retrieval model and efficient control algorithms need be developed to obtain a satisfying performance on a simple hardware bench [17, 18]. In this way, on the one hand we lose accuracy in the phase retrieval model by reduction of the computational complexity; on the other hand we let the model uncertainties be handled by the control algorithm which is based on a feedback principle. 


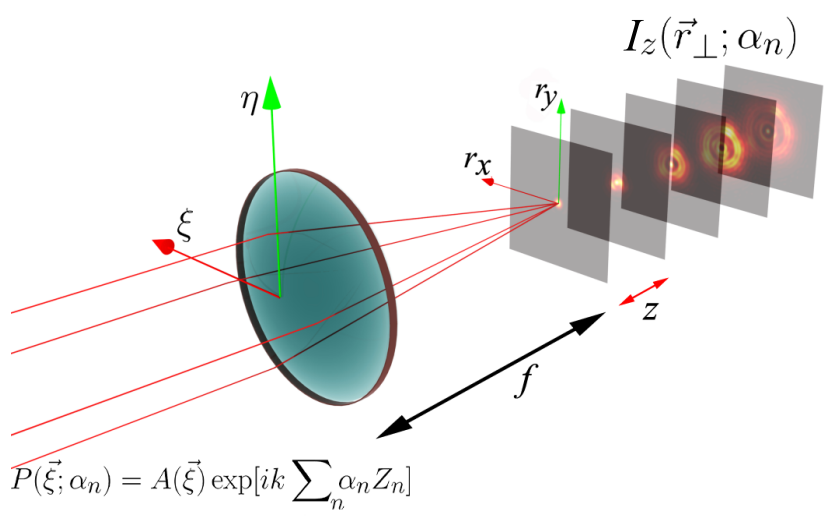

FIG. 1 Graphical representation of an out-of-focus intensity measurement concept.

In particular, in this paper we develop a linear phase retrieval model based on the approximation of small aberrations in the exit pupil function of an optical system. The model is valid for low-numerical aperture (NA) focused and unpolarised optical fields. By choosing correctly the optimal single-plane of measurement and implementing an Iterative Learning Control algorithm (ILC) [19] we are able to perform, on a standard personal computer, fast phase measurements that are suitable for real-time adaptive optics system.

This paper is organised as follows. In Section 2, the linear approximation of the phase retrieval is introduced and the inversion problem is discussed. In Section 3, the control algorithm and the experimental results of the linear phase retrieval in an adaptive optics system are described. Finally, in Section 4, conclusions are drawn.

\section{LINEAIR APPROXIMATION AND PHASE INVERSE PROBLEM}

We analyse the problem by considering the schematic diagram shown in Figure 1. Let the complex field distribution at the exit pupil of an optical system be described by:

$$
P\left(\vec{\xi} ; \boldsymbol{\alpha}_{n}\right)=A(\vec{\xi}) \exp \left[i \phi\left(\vec{\xi} ; \boldsymbol{\alpha}_{n}\right)\right]
$$

where, $A(\vec{\xi})$ is the amplitude distribution (assumed to be uniform and constant) at the exit pupil coordinates $\vec{\xi}=(\xi, \eta)$ and $\phi\left(\vec{\xi} ; \boldsymbol{\alpha}_{n}\right)=\sum_{n}^{N} \boldsymbol{\alpha}_{n} Z_{n}(\vec{\xi})$ is the phase distribution (in radians) described by a superposition of $N$ Zernike polynomials with coefficients $\boldsymbol{\alpha}_{n}$ [20]. Under the condition of small aberrations (e.g., phase variance less than $0.5 \mathrm{rad}$ [21]-[23]), we expand the exponential in Eq. (1) in a Taylor series up to the first order around $\phi=0$ :

$$
P\left(\vec{\xi} ; \boldsymbol{\alpha}_{n}\right) \simeq A(\vec{\xi})\left[1+i \phi\left(\vec{\xi} ; \boldsymbol{\alpha}_{n}\right)\right]
$$

Assuming no polarisation effects and low-numerical aperture (NA), we define $U_{z}\left(\mathbf{r}_{\perp} ; \boldsymbol{\alpha}_{n}\right)$ as the field in the plane $\mathbf{r}_{\perp}=\left(r_{x}, r_{y}\right)$ perpendicular to the optical axis at a distance $z$ from the focus $f$ [24]:

$$
U_{z}\left(\mathbf{r}_{\perp} ; \boldsymbol{\alpha}_{n}\right) \simeq G_{z}[A(\vec{\xi})]+i G_{z}\left[A(\vec{\xi}) \phi\left(\vec{\xi} ; \boldsymbol{\alpha}_{n}\right)\right]
$$

where $G_{z}[\cdot]^{1}$ is the operator that maps the pupil function to the field $U_{z}$. Taking the modulus squared of Eq. (3) and neglecting the quadratic phase term we obtain:

$$
\begin{aligned}
\mathbf{I}_{z}\left(\mathbf{r}_{\perp} ; \boldsymbol{\alpha}_{n}\right)= & \left|U_{z}\left(\mathbf{r}_{\perp} ; \boldsymbol{\alpha}_{n}\right)\right|^{2} \\
\simeq & \left|G_{z}[A(\vec{\xi})]\right|^{2} \\
& +2 \operatorname{Re}\left\{i G_{z}[A(\vec{\xi})] G_{z}\left[A(\vec{\xi}) \phi\left(\vec{\xi} ; \boldsymbol{\alpha}_{n}\right)\right]^{*}\right\} .
\end{aligned}
$$

Here, $\mathbf{I}_{z}\left(\mathbf{r}_{\perp} ; \boldsymbol{\alpha}_{n}\right)$ is the intensity distribution computed at the out-of-focus position $z,\left|G_{z}[A(\vec{\xi})]\right|^{2}$ is the aberration-free PSF at the out-of-focus position $z$, and the final term at the righthand of Eq. (4) that is linear in $\phi$, is the first order perturbation term in the intensity due to the small aberration function $\phi\left(\vec{\xi} ; \alpha_{n}\right)$. For simplicity of notation, we rewrite Eq. (4) using a matrix formalism:

$$
\mathbf{I}_{z}=L_{z} \boldsymbol{\alpha},
$$

where $\mathbf{I}_{z} \in \mathbb{R}^{M}$ is the column vector of the measured intensities ( $M$ is the total number pixels in the detector), $L_{z} \in \mathbb{R}^{M \times N}$ is the matrix of the linear model, and $\alpha \in \mathbb{R}^{N}, \boldsymbol{\alpha}=\left[\alpha_{1} ; \ldots ; \alpha_{N}\right]$ is the column vector of the Zernike coefficients. Therefore, a linearized relation is established between the phase coefficients $\boldsymbol{\alpha}_{n}$ and the intensity distribution $\mathbf{I}_{z}$ in the focal region. Considering that noise is always present in the measured data ${ }^{2}$ and $M>N$, we solve the linear system of equations (Eq. (5)) in a least-square sense. Assuming that $L_{z}$ has full column rank, the least squares solution is given by [25]:

$$
\hat{\boldsymbol{\alpha}}=\left(L_{z}^{T} L_{z}\right)^{-1} L_{z}^{T} \mathbf{I}_{z}
$$

where $\hat{\boldsymbol{\alpha}}$ is the least-squares estimate of the phase Zernike coefficients. It has already been noted in the introduction that the out-of-focus distance can be chosen to be optimal for the phase retrieval problem. According to our previous results in [16] and the results achieved in [13]-[15] , we set the out-offocus distance equal to $u=4 \pi$ (the dimensionless optical unit $u=2 \pi z / \lambda \mathrm{NA}^{2}$ is adopted here [26]). Throughout the paper $\mathbf{I}_{z}$ in Eq. (6) will be the intensity distribution measured at this optimal distance.

\section{CLOSED-LOOP AO RESULTS}

In this section the implementation of the linear phase retrieval from the optimal measurement plane in an adaptive optics setup is described. The sketch of the experimental setup is shown in Figure 2. We use a coherent laser source working at the wavelength of $\lambda=638 \mathrm{~nm}$. The light is coupled by a single mode fiber (assumed to be, in a good approximation, a point source) and then collimated by the lens $L 1$ (focal distance $f_{1}=100 \mathrm{~mm}$ ). A beam splitter (BS) directs half of the beam to a deformable mirror (DM) (Adaptica Saturn 48 actuators push-pull deformable mirror [27]) used as the active element of the AO system. The relay system consisting

\footnotetext{
${ }^{1}$ Here we use the scalar angular spectrum propagation: $G_{z}[\cdot]=$ $\mathcal{F}^{-1}\left\{\mathcal{F}\left[\exp ^{i \frac{k}{2 f} r_{\perp}}\right] \mathcal{F}[\cdot] \exp ^{i k_{z} z}\right\} / i \lambda f$ where $k_{z}=\sqrt{\vec{k}^{2}-k_{x}^{2}-k_{y}^{2}}$ is the $z$ component of the wave vector $\vec{k}$.

${ }^{2}$ for simplicity of notation we do not include the measurement of noise in the equations. The procedure can be improved by modelling the noise and constraining the norm of the solution by a regularisation parameter to improve the conditioning of the problem.
} 


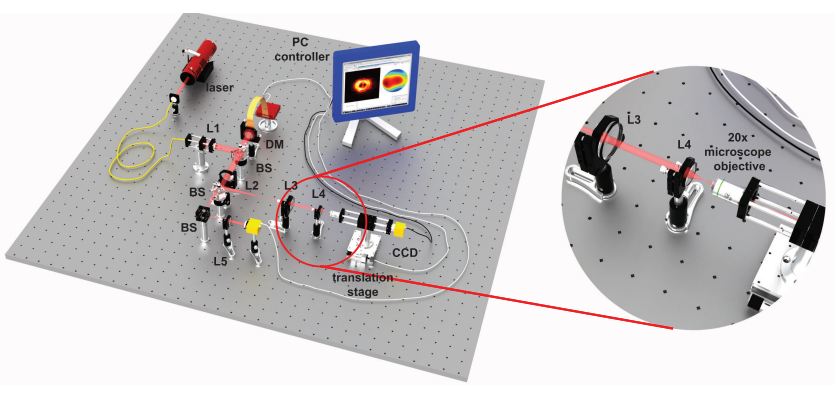

FIG. 2 Sketch of the experimental setup

of the lenses $L 2$ and $L 3$ (with focal length $f_{2}=250 \mathrm{~mm}$ and $f_{3}=100 \mathrm{~mm}$ respectively) ensures the optical conjugation of the pupil of the DM with the back focal plane of the focusing lens $L 4$ (aspheric lens NA $=0.15$ ). The out-of-focus intensity is recorded by a CCD camera (SVCam-eco204) using a 20x microscope objective. These last two elements are mounted on top of a computer-controlled motorised linear translation stage (Physik Instrumente M-014.D01) in order to measure the intensity automatically. An additional relay system (lenses L2 and $L 5$ ) is used to measure the uniformity of the intensity distribution on the mirror pupil which is assumed to be constant in our model.

It should be remarked here that the response of each actuator of our DM is a non-linear function of the driving voltage. Moreover, as a consequence of the continuous (i.e., nonsegmented) surface of the DM, coupling between adjacent actuators of the DM is relatively strong. Those factors make the task of correcting the measured aberrations $\hat{\alpha}$ by the DM more challenging. In particular, these nonlinearities of the DM imply that a solution of the driving voltages should be found iteratively, at the expense of real-time phase correction. In order to overcome these difficulties an ILC algorithm has been implemented in the AO setup. The ILC algorithm is based on a linearised model of the DM described as follows:

$$
\alpha=F \mathbf{V}
$$

where $\alpha \in \mathbb{R}^{\mathbb{N}}$ is the wavefront produced by the surface deformation of the DM, $F \in \mathbb{R}^{N \times 48}$ is the linear model of the $\mathrm{DM}$ and $\mathbf{V} \in \mathbb{R}^{48}$ is the vector of the driving voltages. A detailed description of this algorithm can be found in [28, 29]. In these papers it has been shown that the ILC algorithm outperforms other control algorithms described in the literature and assures convergence to a relatively small steady state of the wavefront error. For completeness of presentation, in the following section we briefly summarise the ILC algorithm.

\subsection{Iterative learning control}

Let $t \in \mathbb{N}$ be a discrete control iteration. The measured wavefront resulting from Eq. (6) at the control iteration $t$ will be denoted by $\hat{\boldsymbol{\alpha}}_{t}$. Therefore we define the wavefront error as:

$$
\mathbf{e}_{t}=\alpha_{d}-\hat{\boldsymbol{\alpha}}_{t}=\alpha_{d}-F \mathbf{V}_{t}
$$

where $\alpha_{d}$ is the vector of the Zernike coefficients of the desired shape wavefront and $\mathbf{V}_{t}$ is the voltage vector at the control iteration $t$. The wavefront error at the control iteration $t+1$ is

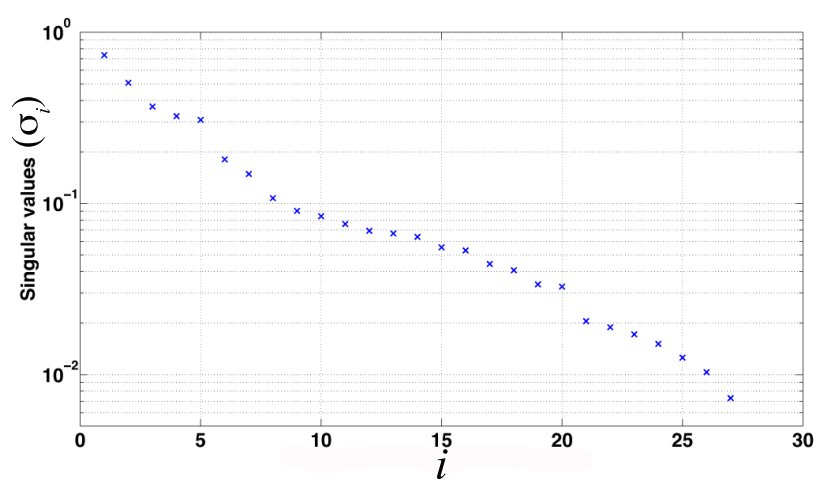

FIG. 3 Singular Values of the mirror's influence function $F$.

given by:

$$
\mathbf{e}_{t+1}=\boldsymbol{\alpha}_{d}-F \mathbf{V}_{t+1}=\mathbf{e}_{t}+F \mathbf{V}_{t}-F \mathbf{V}_{t+1}=\mathbf{e}_{t}-F \Delta \mathbf{V}_{t}
$$

where $\Delta \mathbf{V}_{t}=\mathbf{V}_{t+1}-\mathbf{V}_{t}$ is the voltage control update. The goal of the ILC algorithm is to minimise the wavefront error by solving the following optimisation problem:

$$
\begin{aligned}
& \min _{\Delta \mathbf{V}_{t}}\left\{\left\|\mathbf{e}_{t+1}\right\|_{2}^{2}+\beta\left\|\Delta \mathbf{V}_{t}\right\|_{2}^{2}\right\} \\
& =\min _{\Delta \mathbf{V}_{t}}\left\{\left\|\mathbf{e}_{t}-F \Delta \mathbf{V}_{t}\right\|_{2}^{2}+\beta\left\|\Delta \mathbf{V}_{t}\right\|_{2}^{2}\right\} .
\end{aligned}
$$

Here $\beta$ is a regularisation parameter which penalises $\Delta \mathbf{V}_{t}$ and accounts for the convergence of the control algorithm and $\|\cdot\|_{2}^{2}$ is a 2-norm [25]. From Eq. (10) the ILC control law is obtained as:

$$
\mathbf{V}_{t+1}=\mathbf{V}_{t}+\left(F^{T} F+\beta I\right)^{-1} F^{T} \mathbf{e}_{t} .
$$

Hence, the solution of Eq. (11) at the control iteration $t+1$ is based on the knowledge of the measured wavefront $\hat{\alpha}_{t}$ at the control iteration $t$.

\subsection{Wavefront correction convergence}

In our experiment we attempt to correct for all aberrations that are present in the system. These aberrations are originated by e.g., misaligned components, initial non-flatness of the DM surface, and/or manufacturing imperfections of the optical components. The control algorithm corrects for $N=20$ Zernike coefficients (i.e., 6 Zernike orders, piston omitted, according to Noll notation [20]). We should note here that the maximum number of aberration modes that can be corrected is limited by the number of actuators of the DM and their configuration. This can be estimated by performing a singular value decomposition (SVD) of F. Figure 3 shows that a fall off in the singular values occurs after the $20^{\text {th }}$ singular value. This implies that high order Zernike modes are not reproducible with the DM used in our setup [30]. In Eq. (11) we chose $\beta=0.01$ which has demonstrated, during the experimental data acquisition, to be a good compromise between speed and stability of the control algorithm. The experimental results of the closed-loop wavefront correction are shown in Figure 4 . We observe that, at the control iteration $t=1$, the intensity distribution at the out-of-focus distance of $u=4 \pi$ is clearly affected by aberrations of the system. The estimated phase distribution in this case exhibits a wavefront RMS of $0.059 \lambda$. In our setup, the time between two control iterations $(t$ and $t+1)$ is on average $1.5 \mathrm{~s}$. This relatively large time has 
$t=1$

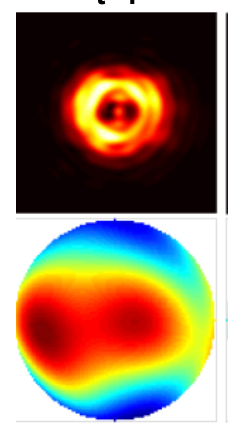

RMS $0.059 \lambda$ $t=2$
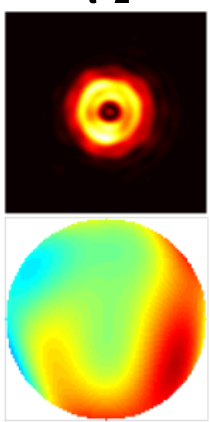

RMS $0.033 \lambda$ $t=3$

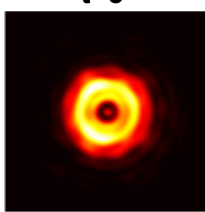

RMS $0.013 \lambda$ $t=5$
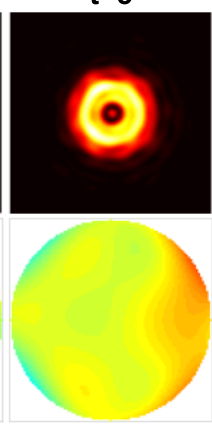

RMS $0.011 \lambda$

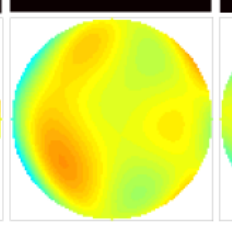

FIG. 4 Time evolution of defocus PSF $(u=4 \pi)$ for different control iterations $t$ (top row) and associated wavefront measurements evolution (bottom row).

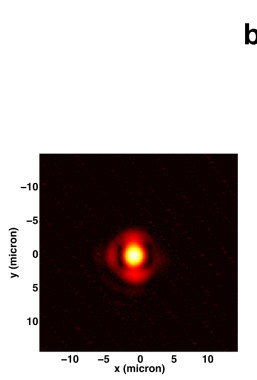

a)
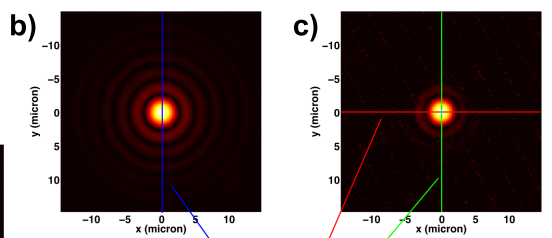

d)

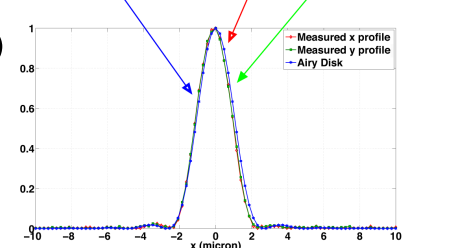

FIG. 5 Intensity measurements of the focal PSF. (a) measured PSF before correction; (b) simulated aberration-free PSF; (c) measured PSF after correction (control iteration $t=13$ ); (d) $x$ and $y$ profiles comparison of the simulated aberrationfree PSF and the measured one.

to be attributed to the slow response of our hardware and the acquisition time of the CCD camera. The time needed for evaluating $\mathbf{V}_{t+1}$ from Eq. (11) is about $0.2 s$ which is relatively fast in comparison to our hardware (for comparison, a non-linear phase retrieval algorithm implemented on the same hardware would need a couple of minutes to estimate the phase measurement). The result in Figure 4 shows that, after the third iteration, the algorithm is able to correct most of the aberrations in the system, achieving a RMS of $0.013 \lambda$. In the successive iterations the algorithm quickly reaches its steady state achieving a final wavefront RMS of $0.008 \lambda$ at the control iteration $t=13$. The quality of the wavefront is also confirmed by the shape of the out-of-focus intensity distribution after convergence. Here we note that the intensity distribution has recovered most of its ideal features i.e., it exhibits a circular symmetry and a minimum intensity at the center. Nevertheless, minor deviations are still noticeable with respect to the ideal aberration-free intensity distribution. As mentioned before, they are attributed to phase aberrations which involve Zernike modes that are beyond the correction capability of the DM used in the experiment.

For completeness, we also measure the intensity distribution of the PFS at the focal plane $u=0$. Figure 5(a) shows the focal PSF at the control iteration $t=1$, when no corrections have been applied to the wavefront. Figures 5(b), 5(c) and 5(d) show the comparison between an analytically computed aberration-free PSF and the measured PSF at the focal plane at the control iteration $t=13$. In terms of Strehl ratio (S) we gain a correction from $S=0.88$ at the control iteration $t=1$ to $S=0.99$ at $t=13$. Note that, as in the previous case, the wavefront corrections are computed from the intensity distribution measured at the out-of-focus distance $u=4 \pi$. Similarly to the previous measurement, we can also observe that the focal PSF has recovered its ideal features. In particular, the mismatch between the $x$ and $y$ cross-sections of the measured PSF after the final iteration of the algorithm and the ideal aberration-free PSF is about $2 \%$. This deviation is a consequence of the ill-posedness of the phase retrieval problem at the focal plane $u=0$. As already demonstrated in [16], in the case of focal plane measurement, particular types of aberrations do not contribute significantly to the intensity profile of the PSF, making the problem of aberration retrieval ill-conditioned. Furthermore the effect of a small aberration is a slight distortion of the non-zero order diffraction rings. From Figure 5(c) we see that only the $1^{\text {st }}$ diffraction ring is clearly visible, while the other rings are washed-out by detector noise which decreases the signal-to-noise ratio (SNR) and hides the contributions of the aberrations to the PSF intensity pattern. On the other hand, the intensity distributions at the out-of-focus distance $u=4 \pi$ are able to optimally reveal the effect of the aberrations, partially due to the optimal choice of the measurement distance (which makes the intensity distribution optimally sensitive to the aberrations) and partially due to the improved SNR.

\section{CONCLUSION}

We have developed a linear model for a phase retrieval algorithm in the approximation of small aberrations for the case of a lower-NA focused field. By appropriately choosing the outof-focus measurement distance of the intensity pattern and applying an Iterative Learning Control algorithm, an efficient closed-loop adaptive optics system has been realised. The experimental results show that fast correction of aberrations can be achieved by this procedure. The results demonstrate that a phase retrieval algorithms based on a linear model can be used as a real-time phase determination. Consequently, this 
approach can be used as an attractive alternative to common wavefront sensing techniques used in adaptive optics system.

\section{Acknowledgements}

This research is supported by the Dutch Ministry of the Economic Affairs and the Provinces of Noord-Brabant and Limburg in the frame of the Pieken in de Delta program. We wish to thank N. Doelman from TNO and J. A. Meisner for helpful discussions. The authors are also grateful to R. Horsten, R. Pols and T. Zuidwijk for their technical support.

\section{References}

[1] N. Hubin, and L. Noethe, "Active optics, adaptive optics, and laser guide stars," Science 262, 1390-1394 (1993).

[2] M. J. Booth, "Adaptive optics in microscopy," Philos. T. R. Soc. A. $365,2829-43(2007)$

[3] M. R. Foreman, C. L. Giusca, P. Török, and R. K. Leach, "Phaseretrieved pupil function and coherent transfer function in confocal microscopy,“ J. Microsc. 251, 99-107 (2013).

[4] A. Roorda, F. Romero-Borja, W. Donnelly, H. Queener, T. Hebert, and M. Campbell, "Adaptive optics scanning laser ophthalmoscopy," Opt. Express 10, 405 (2002).

[5] R. J. Zawadzki, S. M. Jones, S. S. Olivier, M. Zhao, B. A. Bower, J. A. Izatt, S. Choi, S. Laut, and J. S. Werner, "Adaptive-optics optical coherence tomography for high-resolution and high-speed $3 \mathrm{D}$ retinal in vivo imaging," Opt. Express 13, 8532-8546 (2005).

[6] S.-W. Bahk, E. Fess, B. E. Kruschwitz, and J. D. Zuegel, "A highresolution, adaptive beam-shaping system for high-power lasers," Opt. Express 18, 9151-63 (2010).

[7] F. Staals, A. Andryzhyieuskaya, H. Bakker, M. Beems, J. Finders, T. Hollink, J. Mulkens, et al., "Advanced wavefront engineering for improved imaging and overlay applications on a 1.35 NA immersion scanner," Proc. SPIE 7973, 79731G-13 (2011).

[8] A. Haber, A. Polo, I. Maj, S. Pereira, H. Urbach, and M. Verhaegen, "Predictive control of thermally induced wavefront aberrations," Opt. Express 21, 21530 (2013).

[9] J. W. Hardy, Adaptive optics for astronomical telescopes (0xford University Press, New York, 1998).

[10] R. A. Gonsalves, "Phase Retrieval," Proc. SPIE 528, 202-215 (1985).

[11] J. R. Fienup, "Reconstruction of an object from the modulus of its Fourier transform," Opt. Lett. 3, 27-29 (1978).

[12] R. A. Gonsalves, "Phase retrieval by differential intensity measurements," J. Opt. Soc. Am. A 4, 166-170 (1987).

[13] J. R. Fienup, J. C. Marron, T. J. Schulz, and J. H. Seldin, "Hubble Space Telescope characterized by using phase-retrieval algorithms," Appl. Optics 32, 1747-67 (1993).

[14] D. J. Lee, M. C. Roggemann, and B. M. Welsh, "Cramer-Rao analysis of phase-diverse wave-front sensing," J. Opt. Soc. Am. A 16, 1005-1015 (1999).
[15] 0. E. Gawhary, A. Wiegmann, N. Kumar, S. F. Pereira, and $\mathrm{H}$. Urbach, "Through-focus phase retrieval and its connection to the spatial correlation for propagating field," Opt. Express 21, 1662-1669 (2013).

[16] A. Polo, S. F. Pereira, and H. Urbach, "Theoretical analysis for best defocus measurement plane for robust phase retrieval," Opt. Lett., 38812 (2013).

[17] C. U. Keller, V. Korkiakoski, N. Doelman, R. Fraanje, R. Andrei, and M. Verhaegen, "Extremely fast focal-plane wavefront sensing for extreme adaptive optics," Proc. SPIE 8447, 844721-844721-10 (2012).

[18] C. S. Smith, R. Marinic, A. J. D. Dekker, M. Verhaegen, V. Korkiakoski, C. U. Keller, and N. Doelman, "Iterative linear focalplane wavefront correction," J. Opt. Soc. Am. Aosa 30, 2002-2011 (2013).

[19] D. A. Bristow, M. Tharayil, and A. G. Alleyne, "A survey of iterative learning control," IEEE Contr. Syst. Mag. 26, 96-114 (2006).

[20] D. Malacara and W. T. Welford, Optical shop testing (John Wiley Sons, Hoboken, 2006).

[21] T. I. Kuznetsova, "On the phase retrieval problem in optics," Sov. Phys. Uspekhi 31, 364-371 (1988).

[22] W. J. Wild, "Linear phase retrieval for wave-front sensing," Opt. Lett. 23, 573-5 (1998).

[23] J. Braat, P. Dirksen, and A. J. E. M. Janssen, "Assessment of an extended Nijboer-Zernike approach for the computation of optical point-spread functions," J. Opt. Soc. Am. A 19, 858-870 (2002).

[24] J. W. Goodman, Introduction to Fourier optics (Roberts and Company Publishers, Englewood, 2005).

[25] M. Verhaegen and V. Verdult, Filtering and system identification: a least square approach (Cambridge University Press, Cambridge, 2007).

[26] M. Born, and E. Wolf, Principles of optics: electromagnetic theory of propagation, interference and diffraction of light (Cambridge University Press, Cambridge, 1999).

[27] Adaptica Srl, Saturn user manual, http://www.adaptica.com/site/ en/pages/saturn.

[28] A. Polo, A. Haber, S. F. Pereira, M. Verhaegen, and H. P. Urbach, "An innovative and efficient method to control the shape of pushpull membrane deformable mirror," Opt. Express 20, 27922-27932 (2012).

[29] A. Haber, A. Polo, C. S. Smith, S. F. Pereira, P. Urbach, and M. Verhaegen, "Iterative learning control of a membrane deformable mirror for optimal wavefront correction," Appl. Optics $\mathbf{5 2}$, 2363 (2013).

[30] A. Haber, A. Polo, S. Ravensbergen, H. P. Urbach, and M. Verhaegen, "Identification of a dynamical model of a thermally actuated deformable mirror," Opt. Lett. 38, 3061-3064 (2013). 